\title{
Téoros
}

Revue de recherche en tourisme

\section{Les hyperéquipements du tourisme de Bath à Dubaï}

\section{Gérard Beaudet}

Volume 27, numéro 2, été 2008

Les grands équipements touristiques

URI : https://id.erudit.org/iderudit/1070791ar

DOI : https://doi.org/10.7202/1070791ar

Aller au sommaire du numéro

Éditeur(s)

Université du Québec à Montréal

ISSN

0712-8657 (imprimé)

1923-2705 (numérique)

Découvrir la revue

Citer cet article

Beaudet, G. (2008). Les hyperéquipements du tourisme de Bath à Dubaï. Téoros, 27(2), 5-15. https://doi.org/10.7202/1070791ar d'utilisation que vous pouvez consulter en ligne.

https://apropos.erudit.org/fr/usagers/politique-dutilisation/ 


\section{Les hyperéquipements du tourisme de Bath à Dubaï}

\section{Gérard Beaudet}

Si les humains ont rapidement laissé leur empreinte sur le territoire en y disséminant des réalisations qui témoignent de leurs croyances et de leur rapport au sacré, c'est sans contredit le passage du nomadisme à la sédentarité qui a irrémédiablement engendré l'inscription dans l'espace géographique de constructions destinées à constituer un ancrage permanent de groupes humains et une des expressions de leurs cultures matérielles. Apparue il y a quelque 6000 ans, la ville s'en révèle d'emblée une des matérialisations les plus complexes, tandis que palais, temples, sanctuaires y font figure d'équipements de premier plan. Puis, au gré des millénaires, sont construits ou aménagés ports, canaux, ponts, routes et places fortes qui en jalonnent les tracés, favorisent et facilitent les échanges culturels, économiques et sociaux.

De nos jours, on répartit habituellement les équipements constitutifs de l'établissement humain en équipements infrastructurels et superstructurels. Les premiers concernent les installations nécessaires au bon fonctionnement des sociétés - en particulier en ce qui touche la mobilité des personnes et des marchandises - qui sont déployées au sol ou en sous-sol et, généralement, configurées en réseaux. Ces installations regroupent les routes et les autoroutes, les voies ferrées, les voies maritimes, les ports et les canaux, les installations de transport d'énergie (électricité, pétrole, gaz) et de télécommunications, les services urbains (alimentation en eau, évacuation des eaux usées), ainsi que les constructions, les ouvrages et les bâtiments associés qui permettent le raccordement aux territoires, tels les ponts, les viaducs et les tunnels, les gares ferroviaires et maritimes, les aérogares, les barrages et les centrales électriques, les stations de pompage, les usines de traitement et de filtration des eaux.

Quant aux seconds, ils abritent les usages collectifs, notamment culturels (théâtre, cinéma, maison d'opéra, etc.), cultuels (cathédrale, église, temple, sanctuaire, etc.), sanitaires (hôpital, centre de soins prolongés, etc.), scolaires (école, collège, université, etc.), industriels (manufacture, usine, etc.), commerciaux (centre commercial, grande surface, hôtel, etc.), ludiques (casino, parc à thème, etc.) et sportifs (stade, centre de ski, piscine, etc.). Même si l'on évoque parfois à leur égard le concept de réseau, ces derniers sont d'abord des composantes de premier plan des environnements bâtis qu'ils polarisent ou marquent de manière significative.

Bien que la ressource touristique ne soit pas entièrement constituée de main d'homme - la mer, la montagne, les glaciers, le désert, le Grand Canyon, un vaste panorama, etc. - et que sa mise en valeur ne requière pas nécessairement d'interventions spécifiques, le tourisme n'échappe évidemment pas à la nécessité de s'inscrire dans l'espace en s'articulant à des infrastructures et en distribuant des équipements superstructurels. Mais il le fait d'une manière qui lui est propre puisque, si certains équipements sont touristiques par destination, plus nombreux encore sont ceux qui le sont par appropriation, voire par détournement de vocation. Cela s'explique par le fait que le tourisme, avant d'être une activité, est une valorisation, comme le sont le patrimoine et le paysage. En d'autres termes, l'aménagement touristique ne s'articule pas nécessairement à des équipements dont il serait la principale raison d'être. Le caractère non spécifiquement touristique de ses équipements est même la règle plutôt que l'exception.

II n'en reste pas moins que des lieux à vocation touristique dominante, voire exclusive, ont progressivement vu le jour à compter de la fin du XIXe siècle. Mais, en général, ils constituent moins des équipements en tant que tels que des assemblages passablement complexes qu'on pourrait, par analogie aux hypertextes, qualifier d'hyperéquipements. Dans les dernières décennies du $X X^{e}$ siècle, de tels hyperéquipements consistaient en

un casino de jeux, un golf, un palais des congrès, des salles de spectacles, des hôtels de prestige, des restaurants aux chefs réputés, des domaines réservés aux clubs de vacances de type Club Méditerranée, des galeries marchandes ou rues commerçantes de luxe, des succursales de banques de taille internationale [...], des agences de voyages et des agences immobilières, la palette usuelle des services élaborés pour voyageurs et bagages [...] Dans ces centres, l'affinement va parfois très loin : on aménage un hippodrome, un jardin botanique et zoologique, un grand stade ou plusieurs stades spécialisés susceptibles d'accueillir des équipes de valeur. En pays tempérés, les hôtels de classe doivent offrir des piscines chauffées. En montagne, les pistes de ski ont intérêt à être équipées d'un éclairage nocturne ${ }^{1}[. .$.$] ; d'autres sports sont$ à prévoir pour amplifier l'offre ; des compétitions internationales sont destinées à rythmer le déroulement de la saison. Sur le littoral, le port de plaisance, des marinas, l'éventail complet des sports nautiques sont recherchés. Partout, l'équitation, 
les courts de tennis ont fait leur entrée. (Wackermann, 1988: 13)

Lancé à la fin du siècle dernier afin de diversifier l'économie des Émirats arabes unis, le projet récréotouristique de Dubaï est en voie de faire paraitre bien modeste cette nomenclature. Cette gigantesque station touristique jumelée à ce qui était, jusque dans les années 1970, une petite ville fondée au XVIII siècle, devrait en effet devenir, selon les souhaits de ses promoteurs, une des plus importantes destinations touristiques du monde. Le projet est constitué de trois îles artificielles en forme de palmier inscrites dans de vastes atolls (Palm Islands) et devant abriter plusieurs complexes hôteliers, des milliers de villas et des marinas, d'un archipel de quelque 60 kilomètres carrés de superficie et comportant plus de 250 îlots - dont plusieurs seront voués au tourisme - reproduisant une mappemonde (The World), d'une marina construite sur un bras de mer s'enfonçant sur plus de quatre kilomètres dans le désert et ceinturée de plus de 200 immeubles, ainsi que d'un immense front maritime qui doit notamment accueillir le plus vaste centre commercial de la planète. Parmi les équipements les plus distinctifs de la station, figurent un spectaculaire hôtel en forme de voile de plus de 300 mètres de haut et comptant 202 suites doubles, un complexe sportif de quelque 7,5 kilomètres carrés de superficie et comportant des terrains de golf, de football, de rugby et de cricket, ainsi qu'un centre de ski abrité dans un bâtiment d'une hauteur de 80 mètres et couvrant plus de trois hectares où l'on trouve une piste de ski de 400 mètres, un snowpark (parc de planche à neige) et une piste de bobsleigh, tout cela sans compter les divers parcs à thème et centres d'attractions. Comptant plus de 125 kilomètres de rivages artificiels sur le golfe Persique, la station est par ailleurs desservie par un aéroport international pouvant accueillir 70 millions de passagers par an, auquel s'ajoutera bientôt un second aéroport.

Bien que ce projet s'inscrive sur une lancée qui n'est pas entièrement nouvelle - on peut penser à Las Vegas, dont la transformation en ville touristique s'opère au tournant des années 1930 aux années 1940, au Disney World Resort d'Orlando, inauguré au début des années 1970, ou à Eurodisney, ouvert au début des années 1990 -, il n'en reste pas moins qu'il fait aujourd'hui figure d'exception, tant par sa démesure que par son statut de création ex nihilo. Rarement en effet a-t-on assisté à un tel écart entre l'intérêt relatif de la ressource - en l'occurrence un littoral - et la taille des installations destinées à son exploitation.

Ces hyperéquipements sont l'aboutissement d'une construction progressive qui s'est amorcée avec l'invention du tourisme, au tournant du XVIII ${ }^{e}$ au XIX siècle, et qui en a depuis accompagné le développement. C'est cette construction qu'entend évoquer le présent texte.

\section{Des équipements devenus ressources aux équipements dédiés}

Les premiers touristes empruntent, au gré de leurs pérégrinations, les routes terrestres et maritimes, ainsi que les moyens de transport, qui sont développés à des fins militaires et commerciales. Puis, ils profitent, comme les autres voyageurs, des inventions que multiplie le XIXe siècle dans le but d'accroître et de faciliter la mobilité des personnes et des marchandises. Rapidement, certaines infrastructures deviennent étroitement associées au tourisme. C'est le cas, en particulier, des voies ferrées dont la multiplication, dès avant le milieu du XIX siècle, favorise les déplacements, invite au voyage, voire crée littéralement certaines destinations jusqu'alors fréquentées par quelques initiés. Cette association du train au tourisme s'affirme encore davantage au tournant du XIX au XXe siècle, alors qu'affiches, peintures, romans et autres écrits, de même que cinéma, éventuellement, contribuent à construire et à diffuser l'imaginaire du voyage en train².

Le corridor ferroviaire emprunté par le mythique Orient Express, mis en service en 1883, aura probablement été, de ce point de vue, ce qui se rapproche le plus d'une infrastructure explicitement dévolue au tourisme. Reliant Paris et Constantinople en passant par Strasbourg, Munich, Vienne, Budapest et Bucarest, cette liaison ferroviaire permet en effet la découverte de la marge occidentale d'un Orient nimbé de mystères au terme d'un itinéraire réalisé dans des voitures de grand luxe ${ }^{3}$. Au Canada, le chemin de fer du Canadien Pacifique (C.P.) comporte également d'entrée de jeu une vocation touristique.

Conscient du potentiel attractif des Rocheuses, où vient d'être créé le premier parc national canadien, le directeur de l'entreprise, W.C. Van Horne, soutient que, à défaut de pouvoir exporter les paysages, il entend importer les touristes. Le C.P. ponctue donc, à compter de la fin des années 1888, le corridor ferroviaire qui traverse l'Ouest canadien de gares comportant des salles à dîner et des chambres et construit quelques grands hôtels, dont le Banff Springs Hotel (1886). La découverte par les touristes des beautés sauvages de l'Ouest, favorisée par la diffusion de reportages photographiques - notamment ceux du photographe William Notman -, y est donc contemporaine de la colonisation de ces vastes contrées.

L'invention de la navigation à vapeur joue également un rôle significatif dans le développement du tourisme. Même si les grandes croisières ne sont formellement proposées qu'à compter des années 1890, des compagnies maritimes offrent des excursions sur plusieurs grands fleuves dès les années 1820, dont le Rhin et le Danube, qui jouissent d'une aura romantique. Au Québec, le service des bateaux blancs inauguré dans les années 1840 combine excursions sur le Saint-Laurent et le Saguenay et dessert les principales stations de villégiature du Bas-du-Fleuve et de Charlevoix. Aux États-Unis, le service des célèbres steamboats du Mississippi connaît son apogée de 1870 à 1920, rivalisant même avec le train. Bien que n'affichant généralement pas le panache des gares ferroviaires, les quais et les gares maritimes qui jalonnent ces voies navigables n'en révèlent pas moins l'importance qu'a eu le transport fluvial pour l'essor de plusieurs destinations touristiques, dont les hôtels et les restaurants dotés de terrasses permettent d'apprécier le panorama aussi bien que l'animation fluviale.

À une échelle locale, bon nombre d'ouvrages d'ingénierie rattachés à des équipements infrastructurels sont appropriés par le tourisme. C'est le cas, entre autres, des ponts du Gard (v. 19 av. J.C.), de Saint-Bénézet - mieux connu sous le nom de pont d'Avignon - (1185), du Ponte Vecchio (1345), des 
ponts d'Ironbridge Gorge (1779), de Brooklyn (1883), de Londres - Tower Bridge - (1894) et du Golden Gate à San Francisco (1937), ainsi que des viaducs de Garabit (1884) et de Millau (2004). La renommée de certains d'entre eux est incidemment telle que leur vue suffit à évoquer la ville où ils sont érigés. De nombreux canaux en Europe continentale et en Grande-Bretagne acquièrent aussi une nouvelle vocation au cours des dernières décennies en accueillant des plaisanciers, de plus en plus nombreux à parcourir le pays et à en découvrir les paysages à un rythme qui semblait révolu. Dans le cœur industriel de l'Angleterre, ce recyclage permet au surplus la conservation et la mise en valeur d'une batellerie autrefois vouée au transport des matières premières et des marchandises et conçue spécifiquement pour un réseau de canaux et d'écluses extrêmement étroits ${ }^{4}$. Au Québec, les canaux de Lachine (rouvert à la navigation en 2002 après plus de trente ans d'abandon) et de Chambly ne doivent aujourd'hui leur maintien en service qu'à l'essor de la navigation de plaisance. D'une architecture se revendiquant de celle des plus grands palais, les stations des métros de Moscou (à partir de 1935) et de Saint-Pétersbourg (dans les années 1950) comptent aussi au nombre de ces équipements infrastructurels qui sont devenus de véritables icônes touristiques.

Du côté des équipements superstructurels, on mise d'emblée sur des emprunts et les premiers équipements valorisés sont ceux légués par le monde romain. Parfois intactes, mais la plupart du temps sévèrement abîmées, voire réduites à l'état de ruines, ces constructions monumentales constituaient, depuis la Renaissance, de véritables références, tant chez les lettrés, les artistes et les antiquaires que chez les architectes. Ensevelie à l'occasion de l'éruption du Vésuve en l'an 79, la ville romaine de Pompéi fut découverte en 1748. Son état de conservation, révélé par des fouilles entreprises l'année de sa localisation et étendues à partir de 1770, lui confère, de ce point de vue, un intérêt inégalé, dans la mesure où la découverte de l'entièreté du tissu urbain est, en quelque sorte, le motif du voyage.

Apparu d'abord en Allemagne et en GrandeBretagne au tournant du XVIII e au XIXe siècle, le Romantisme contribue toutefois à élargir le spectre des emprunts en revisitant le Moyen Âge. Cathédrales et monastères, de même que palais et châteaux-forts de l'époque romane et gothique, retiennent graduellement l'attention et valent parfois le détour. En raison de la concentration d'édifices anciens prestigieux qu'elles abritent, en particulier les palais et les églises, plusieurs villes s'imposent par ailleurs à compter des années 1740, dont, au premier chef, Londres, Paris, Vienne et Prague. Mais cet engouement ne tient pas qu'à la présence de vestiges de l'Antiquité et, dans une moindre mesure, du Moyen Âge. II tient aussi à différentes manifestations d'une modernité qui se répand dans toute l'Europe et dont témoignent les hôtels, les cafés et les restaurants qui se multiplient, au bonheur des visiteurs.

Mais, plus encore, cette modernité s'exprime dans un renouveau urbanistique et architectural. Rome aura montré l'exemple. La reconstruction de la basilique Saint-Pierre, entreprise quelques années avant le sac de 1527, constitue, de ce point de vue, un moment inaugural. L'édifice n'est achevé qu'en 1615, au moment où la ville est elle-même transformée en vaste chantier qui voit se multiplier percées et places mettant en scène de nouveaux édifices d'architecture baroque et de nombreuses œuvres d'art public. La transformation est telle qu'un ambassadeur vénitien note, dès le début des années 1620, que les visiteurs montrent presque autant d'intérêt pour les « attractions touristiques modernes » que pour les vestiges de l'Antiquité.

En 1666, le cœur de Londres est rasé par un terrible incendie. Même si les autorités ne parviennent pas à imposer l'ambitieux projet de réaménagement inspiré des précédents romains, la reconstruction de la cathédrale Saint-Paul (1675-1710), combinée à la réalisation de quelques percées et à l'élargissement des rues, modifie de manière significative la physionomie de la ville. À un point tel que Voltaire, dans un texte de 1749 intitulé Des embellissements de Paris, se désolera en ces termes du sort réservé à la capitale française: «Quoi ! Ne sera-ce jamais qu'à cette dernière extrémité [la ruine consécutive au grand incendie de Londres] que nous ferons quelque chose de grand ! Si la moitié de Paris était brûlée, nous la rebâtirions superbe et commode. »Malheureusement

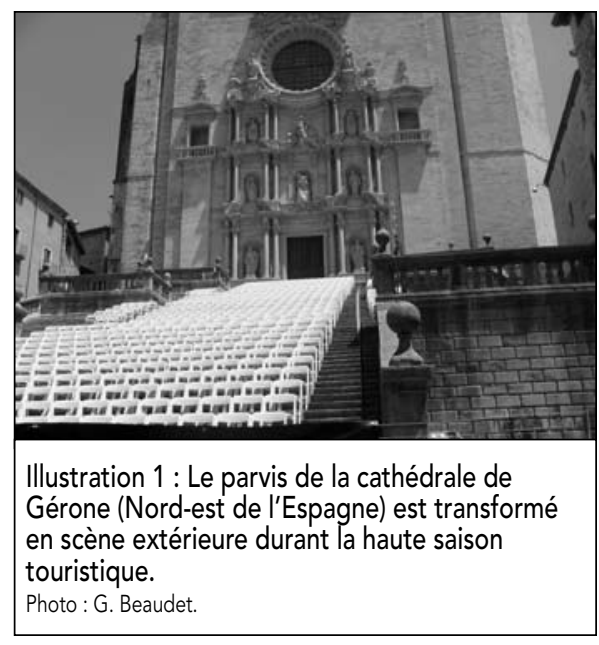

pour l'illustre écrivain, Louis XIV avait préféré se réfugier à Versailles. Quelques années plus tard, Lisbonne est complètement détruite par un tremblement de terre, suivi d'un razde-marée et d'un incendie. Le marquis de Pombal, alors premier ministre, prend les choses en main et entreprend de reconfigurer entièrement le centre de la ville dévastée. Vastes places publiques, larges rues bordées de trottoirs et se coupant à angle droit et bâtiments au goût du jour - raccordés à l'égout - confèrent à Lisbonne une grandeur qui sied bien à son statut de capitale ; ce dont témoignent de nombreux visiteurs.

Venise, que bon nombre de peintres et d'auteurs apprivoisent par le pinceau et la plume, fait aussi partie du nombre des villes que les voyageurs fréquentent, même si elle semble s'être placée en retrait du monde. La Sérénissime doit notamment sa renommée à de nombreux artistes, dont Canaletto, qui y peint ses premiers tableaux vers 1720 . Quelques années avant qu'il ne quitte pour s'installer à Londres, où il séjourne de 1746 à 1753, il met en vente des reproductions d'une dizaine de ses vedute ${ }^{5}$, dont sont particulièrement friands les voyageurs anglais. En raison de l'ampleur de la demande pour de telles vues, Venise est alors considérée comme le principal centre d'activité des védutistes. Mais la ville connaît une éclipse avec la fin de la République (1797). Les voyageurs ne la redécouvrent qu'après la chute de l'empereur Napoléon en 1815. La Cité de la lagune devient une véritable icône d'un tourisme urbain avant la lettre avec l'arrivée du chemin de fer (1846). Le paysagiste Joseph Turner, qui s'y rend à quelques reprises à compter de 
1819, ainsi que de nombreux autres peintres, contribuent significativement à construire et à diffuser la renommée de la ville, par exemple John Ruskin, qui multiplie les croquis de ses principaux édifices et publie son célèbre ouvrage Les pierres de Venise de 1851 à 1853 (Jeancolas, 1999).

Bien que l'architecture vernaculaire suscite encore très peu d'intérêt ${ }^{6}$, la ville de Carcassonne devient également une destination prisée dès les années 1860, au moment où Viollet-le-Duc entreprend les travaux de restauration de l'auguste cité qui, avec sa double enceinte crénelée, ponctuée de nombreuses tours et percée d'imposantes portes, restait l'exemple le plus achevé de l'urbanisme militaire médiéval. D'autres villes se démarquant par leurs sites spectaculaires, à l'instar d'Édinbourg, bénéficient aussi de cet engouement nouveau, même si les conditions sanitaires y sont parfois déplorables. Québec suscite un tel engouement, notamment auprès de riches Étatsuniens qui croient découvrir une ville médiévale. Cette confusion sera d'ailleurs entretenue dans les années 1870, au moment où l'enceinte est sauvée de la démolition par le gouverneur général de l'époque, Lord Dufferin, qui est également l'instigateur de la reconstruction des portes dans un esprit romantique - et dans un style évoquant le Moyen Âge - et de nombreux aménagements destinés à l'agrément des résidents autant qu'au plaisir des touristes, déjà passablement nombreux. ॥ faut toutefois attendre 1893 pour assister à l'inauguration du château Frontenac sur le site retenu par Dufferin pour l'implantation d'un grand hôtel' .

La campagne d'Égypte de Napoléon Bonaparte (1798-1799), la consolidation des grands empires coloniaux d'Afrique du Nord, du Proche et du Moyen-Orient, des Indes et d'Indochine dans la première moitié du XIX siècle, de même que l'accession à l'indépendance de la Grèce (1830), avaient entretemps favorisé la multiplication des voyages dans des contrées jusque-là fréquentées surtout par des missionnaires et des commerçants, ainsi que la publication de récits de voyage, dont le Voyage dans la haute et basse Égypte pendant les campagnes du général Bonaparte du baron Dominique Vivant Denon, paru en 1802, constitue en quelque sorte le prototype. Mais ces événements auront aussi favorisé la révélation de nouvelles richesses, notamment à la faveur de la multiplication des découvertes et des fouilles archéologiques. Les vestiges de temples, de sanctuaires, de palais et de villes - dont ceux de Karnak en 1798, de Pétra en 1812, d'Abou Simbel en 1817 et d'Angkor Vat en 1861 - suscitent un engouement qui incite au voyage. Les conditions dans lesquelles celui-ci s'effectue restent toutefois difficiles, voire périlleuses, et il faudra attendre la fin du siècle pour que le nombre de touristes désireux de visiter ces sites augmente significativement. Plusieurs autres sites majeurs auront alors été découverts ${ }^{8}$.

Le $X X^{e}$ siècle tarde à relancer cette expansion de la mise en tourisme des équipements du passé. L'héritage laissé par l'ère victorienne ne trouve guère de défenseurs parmi les esprits éclairés et les amateurs du passé. Est-il utile de rappeler que la tour Eiffel, érigée à l'occasion de l'exposition universelle de 1889, en avait offusqué plus d'un ? Quant à la reconstruction des cœurs historiques de plusieurs villes du continent entreprise dès avant la fin de la Première Guerre mondiale, elle a certes suscité des débats entre les tenants de la reconstitution à l'identique et ceux d'une modernisation. L'enjeu touristique ne semble toutefois pas avoir été déterminant, sauf dans le cas des principaux monuments. II faut attendre les années 1960 pour voir émerger un intérêt pour ces centres historiques, même si les nombreuses reconstructions consécutives à la Seconde Guerre mondiale sont l'objet de prises de position encore plus tranchées qu'elles ne l'avaient été trente ans plus tôt.

Protégés à titre de secteurs sauvegardés (France), d'arrondissements historiques (Québec), de conservation areas (GrandeBretagne), d'historic districts et d'urban cultural parks (États-Unis) ou en vertu d'autres statuts, ces centres historiques sont en général scrupuleusement restaurés et accueillent, pour la plupart, des activités et des services touristiques passablement denses, souvent au détriment des résidents et des services de proximité qui ne sont pas destinés en priorité aux visiteurs.

Puis, au milieu des années 1970, on assiste à une entrée en force du monde industriel dans le champ du patrimoine et, ce faisant, dans celui du tourisme. Manufactures, centrales thermiques, ouvrages d'art, vieux équipements portuaires et ferroviaires et installations diverses laissés à l'abandon par une désindustrialisation, qui multiplie les friches urbaines et périurbaines, mobilisent experts et citoyens, d'abord en Grande-Bretagne, puis dans l'ensemble des pays occidentaux. ॥ en est de même des nombreux bâtiments publics caractéristiques de l'âge du fer et de la fonte, dont les halles de marché et les gares ferroviaires.

Dans la plupart des villes, le réaménagement des vieux fronts portuaires, l'installation de musées et d'autres équipements culturels dans d'anciens bâtiments industriels, le développement d'une muséologie spécifiquement adaptée aux particularités des vastes complexes de production, favorisent la venue de touristes. Plusieurs de ces réalisations - notamment le musée d'Orsay à Paris (une ancienne gare), la New Tate Gallery de Londres (une ancienne centrale thermique), le centre d'interprétation de Lowell au Massachusetts (une ancienne manufacture), le Vieux-Port de Montréal - acquièrent incidemment une grande renommée et contribuent de manière significative à l'attractivité des lieux.

II n'y a toutefois pas que les installations industrielles désuètes qui entrent dans les circuits du tourisme. Alimenté tant par le déclin des productions manufacturières dans nos économies fortement tertiarisées que par la volonté de pérenniser certaines productions qui ont significativement marqué des villes ou des régions ou encore de valoriser de nouvelles filières industrielles - par exemple l'électronique, le solaire ou l'éolien -, un tourisme en entreprises (work watching) et un tourisme technologique - centrale hydroélectrique, éoliennes, etc. - se sont en effet développés depuis la fin des années 1980 (Gelbman, 2007).

Un peu partout, des villages, des bourgs et des petites villes maintenus dans l'ombre depuis le développement spectaculaire des métropoles et des capitales sont redécouverts au cours des dernières décennies. Si ce sont la plupart du temps, en contexte européen, des bourgades et des villes de l'ère préindustrielle, ce sont plutôt, de ce côté-ci de l'Atlantique, 
des villages et des villes de l'époque de la première révolution industrielle. Passablement épargnés par les grands bouleversements des années d'après-guerre, ils présentent souvent une homogénéité qui contraste avec le caractère hétéroclite des vastes périmètres engendrés par l'étalement urbain. Ils incarnent ces voisinages familiers qui se sont passablement érodés dans les villes moyennes et grandes et ils constituent aujourd'hui des lieux d'ancrage privilégiés d'un tourisme culturel qui cherche à offrir une alternative à ce que proposent les grands centres urbains.

Quant à la modernité définie par les architectes et les urbanistes dès les premières décennies du XXe siècle, elle a tardé à s'inscrire à l'ordre du jour de ces conversions. Les efforts consentis par les édiles de la ville du Havre en faveur de l'inscription sur la liste du patrimoine mondial de l'UNESCO' (2005) de cette œuvre majeure de reconstruction de l'illustre Auguste Perret, puis de sa promotion à titre de destination touristique, montrent incidemment que cette conquête de la modernité par le tourisme reste embryonnaire. II s'agit de comparer le destin de cette reconstruction moderniste avec celui de Saint-Malo, reconstruite quant à elle "à l'identique », pour s'en convaincre.

L'émergence de la modernité dans l'univers touristique repose plutôt sur la construction des grands équipements constitutifs des stations balnéaires et de montagnes, dont la plupart ont été érigés après la Seconde Guerre mondiale. Au Québec, le complexe architectural que le baron Empain fait ériger à Sainte-Marguerite-du-Lac-Masson dans les années 1930 en constitue un exemple aussi exceptionnel que précoce. Le front de mer de style Art déco construit à compter de la fin des années 1920 à Miami est aussi du nombre. Mais il n'y a là rien de comparable à ce que proposent les grandes stations des années 1960 et 1970, dont La GrandeMotte sur le littoral languedocien, la Plagne et La Plagne Bellecôte en Savoie ou Albena (Bulgarie) sur la mer Noire constituent des exemples renommés. Seule la station balnéaire de Prora, érigée sur la Baltique à l'époque de la consolidation de l'Allemagne nazie, anticipait réellement ce mouvement. Les huit imposants bâtiments résidentiels construits entre 1936 et 1939 et s'étirant sur plus de quatre kilomètres de littoral devaient en effet accueillir quelque 20000 vacanciers, auxquels étaient destinés plusieurs équipements collectifs, dont des piscines, un théâtre, un cinéma et une salle de plus de 20000 places. Bien que le programme d'équipements soit resté inachevé, la station n'en annonçait pas moins les vastes opérations littorales qui seraient menées durant les Trente Glorieuses en Europe de l'Ouest.

L'intérêt marqué des touristes du XIX siècle pour des bâtiments isolés et des ensembles monumentaux, dont quelques rares centres historiques, n'empêche toutefois pas le développement d'un autre type de destinations touristiques. Confrontés à l'insalubrité qui règne dans la plupart des villes - bon nombre de beaux quartiers compris - et mobilisés par les promesses de l'hygiénisme, les membres des familles royales et de l'aristocratie répondent favorablement à la découverte, au tournant du XVIII ${ }^{e}$ au XIXe siècle, des vertus sanitaires et curatives des eaux de source, ainsi que de l'eau de mer et de l'air salin.

\section{Les équipements d'un tourisme urbain pratiqué à l'écart de la ville}

Le tourisme a partie liée avec la ville. Déjà, à l'époque du Grand Tour, cette dernière est étape obligée au terme de déplacements quotidiens sujets à grands et petits désagréments. Mais peu de richesses méritent le détour. On n'en a longtemps que pour Rome, dont les grands monuments de l'Antiquité - le Forum, le Colisée, les Thermes et le Panthéon - sont des incontournables. Puis, peu à peu, d'autres villes ponctuent les itinéraires, notamment Orange, Nîmes et Arles dans le sud de la France, où l'on peut admirer arcs de triomphe, portes, théâtres, arènes et temples dans un état de conservation variable. Dans les autres villes-étapes, on se contente d'apprécier les réalisations de l'urbanisme classique et les jardins des palais. Le tourisme n'a alors pas encore produit d'équipements qui lui seraient propres ; il vit essentiellement d'emprunts. II revient aux Anglais de changer la donne. Et le monde romain est encore au rendez-vous.

\section{Bath et l'essor des villes d'eaux}

À l'orée du XVIII siècle, Bath était une petite bourgade réputée pour ses industries du lainage. Mais elle abritait en son centre un complexe de bains datant de l'époque romaine - d'où son nom d'Aquae Sulis -, qui avait fait la renommée de cette ville qui comptait parmi les principales stations thermales de l'Empire d'Occident. Si les bienfaits de ses eaux avaient continué à être appréciés après la chute de Rome, la petite cité n'en avait pas moins sombré dans une certaine léthargie. Au XVII siècle, l'intérêt renouvelé pour la valeur curative des eaux de source favorise toutefois l'arrivée de médecins, la construction de nouveaux bains et l'ouverture d'auberges destinées aux curistes. Quoique difficile d'accès, la ville s'impose rapidement auprès de l'aristocratie.

Les ingrédients d'un success story se mettent en place à compter du début du XVIII e siècle. Si l'érection du Royal Mineral Water Hospital (1738) confirme la valeur curative des eaux, c'est sans contredit la construction à l'emplacement des sources des bâtiments et des installations destinés à agrémenter le séjour des visiteurs qui confère à la ville son caractère exclusif. Assembly Rooms (1769-1771), Hot Bath (1773-1777) et Pump Room (1795) donnent en effet aux ablutions et à la consommation de l'eau un cadre qui favorise les divertissements mondains. Mais le caractère innovateur et l'exemplarité de Bath ne s'arrêtent pas là. La ville elle-même est en effet conçue comme un équipement (Cossic, 2003). Puisant au répertoire de l'urbanisme et de l'architecture géorgiens, promoteurs et concepteurs façonnent une ville nouvelle entièrement vouée au bien-être des nantis. Si Queen Square (1728-1735), The Circus (1754-1768) et Royal Crescent (1767-1775) en constituent les composantes résidentielles les plus remarquables, plusieurs autres réalisations contribuent à faire du cadre urbain un espace de mise en scène dont les résidents et les visiteurs sont les acteurs.

Malgré la desserte ferroviaire et l'amélioration du réseau routier, l'effet de mode s'estompe au milieu du XIX siècle. Accessible par train à compter de 1841, la station balnéaire de Brighton déclasse définitivement Bath. L'influence exercée par ce qui fut de toute évidence la première ville essentiellement touristique d'Europe aura néanmoins été considérable. 
Bath a en effet créé le modèle de la station thermale dont on s'inspire ailleurs. En Europe des dizaines de villes d'eaux se développent. Plusieurs sources thermales sont redécouvertes aux $X V^{e}$ et $X V^{l}$ siècles. Mais c'est au début du XIXe siècle que la plupart d'entre elles sont prétexte à la construction de véritables petites villes essentiellement dévolues à l'accueil des visiteurs de marque. En Angleterre, Royal Tunbridge Wells et Buxton (Devonshire) sont les émules de Bath. Sur le continent, Baden-Baden - surnommée le Paris allemand ou la Capitale d'été de l'Europe -, Baden bei Wien - aussi connue sous le nom de la petite Vienne -, Bad Ischl, Spa - qualifiée de Café de l'Europe -, Budapest - la ville des bains -, Montecatini, Marienbad, Karlsbad, Aix-les-Bains, Évian-les-Bains, Vichy - la reine des villes d'eaux - et Eugénieles-Bains sont les plus connues (Saurat, 1999). Buvettes, galeries, thermes, casinos, palaces, salles de concert, parcs et jardins, tous plus somptueux les uns que les autres, y accueillent, à compter de la fin du XVIII siècle, les têtes couronnées et les aristocrates de toute l'Europe et parfois aussi d'ailleurs.

On compte également plusieurs stations thermales en Amérique du Nord. Dans l'État de New York, Saratoga Springs s'impose, tant par son cadre bâti que par sa fréquentation - la station compte plus de 10000 lits au début des années 1880 -, à l'égal des hauts lieux du thermalisme européen. Au Canada, Caledonia Springs - fondée en 1835 - devient rapidement le rendez-vous du monde politique et de la bourgeoisie industrielle. Mais, ici comme en Europe, le déclenchement de la Première Guerre mondiale accélère le déclin du thermalisme tel qu'on l'avait connu depuis le tournant du XVIII au $X I X{ }^{e}$ siècle. Certaines destinations sont plus sévèrement affectées que d'autres. C'est le cas de Bath (Lambert, 2001).

En première moitié du siècle dernier, le passage des ans, combiné à la négligence et à l'insouciance des propriétaires et des autorités, y menace l'intégrité des cadres bâtis. Des campagnes de sauvegarde sont lancées dès le début du XXe siècle et des mesures de protection du patrimoine sont adoptées. Rien n'est toutefois définitivement acquis. Le cadre bâti subit en effet d'importants dommages durant la Seconde Guerre mondiale.
Plans de reconstruction et démolitions massives constituent, au lendemain du conflit, une menace encore plus sérieuse. Les efforts de protection s'intensifient et la ville est finalement inscrite sur la liste du patrimoine mondial de l'UNESCO en 1987. Aujourd'hui, mise en valeur du patrimoine, piétonisation d'une partie du centre historique et inauguration des installations thermales du Thermae Bath Spa (2006) contribuent à refaire du cœur de la ville un véritable équipement touristique.

Saratoga Springs, tout comme Bath, connait une certaine éclipse de popularité au début du siècle dernier. Reconnue à titre de parc culturel urbain, la petite ville de NouvelleAngleterre vit également une renaissance spectaculaire au cours des dernières décennies, comme la plupart des villes d'eaux désormais considérées comme patrimoines nationaux. D'autres stations en revanche, comptant souvent parmi les plus modestes, connaissent, à l'instar de Caledonia Springs, une fin abrupte.

\section{Les stations balnéaires et de montagne}

Bénéficiant de l'intérêt que lui porte la famille royale britannique au milieu du XVIII' siècle, la petite ville de Brighton devient rapidement la rivale de Bath. Archétype de la station balnéaire, elle n'impose que progressivement la mode du séjour au bord de la mer. En effet, la deuxième moitié du siècle est davantage propice à la fréquentation hors saison de la montagne et du littoral, la fraîcheur estivale de la première et le climat tempéré des mois d'hiver du second justifiant les pérégrinations de quelques-uns. C'est que le bain de mer n'attire pas encore les foules. Cautionnée par la médecine, qui compose avec la morale puritaine de l'époque et permet petit à petit de surmonter la crainte millénaire des immensités océanes, l'immersion conserve un caractère curatif. La baignade ne s'émancipe que lentement de la supervision médicale et morale.

La fréquentation du littoral ne requiert en conséquence pas de vastes projets d'équipements, du moins jusqu'au milieu du siècle suivant. Tout au plus préside-t-elle à la construction de résidences secondaires, particulièrement sur la côte méditerranéenne, où les premières implantations consistent habituellement en villas distribuées au voisinage des hameaux et des petites villes, souvent sur des hauteurs qui permettent d'apprécier les vues et de profiter de l'air du large. Mais la renommée de certains sites se propage rapidement, notamment à la faveur de la diffusion des œuvres des peintres, des graveurs et des littéraires qui les valorisent. Si certains développements s'improvisent au gré des arrivées, d'autres sont l'objet d'attentions particulières. Comme à Bath, bien que dans une mesure moindre, l'influence du monde romain joue, en particulier dans le sillage de la publication, en 1764, de l'ouvrage de Robert Adam, Ruins of the Palace of the Emperor Diocletian at Spalatro in Dalmatia, écrit à la suite des inventaires réalisés entre 1754 et 1758. L'imposant complexe architectural construit sur le littoral de l'Adriatique au tournant du III au IV siècle marque fortement l'imaginaire de ses découvreurs et influence les créations balnéaires de la seconde moitié du XIX siècle. II aura toutefois fallu plus d'un siècle de gestation avant d'en arriver à la création d'équipements de cette envergure.

À Brighton, les premiers établissements thermaux se dressent au voisinage du village de pêcheurs à compter de 1760. Un terrain vague utilisé pour le séchage des filets (The Old Steine) est transformé en pelouses et en promenades à partir de 1780. La construction du Royal Pavillon, en 1787, inaugure l'aménagement d'un important corridor vert qui structure, dans le prolongement du Old Steine, le cœur de la station. Découverte en 1763 par l'écrivain britannique Tobias Smollett, la petite ville de Nice devient rapidement une villégiature recherchée par les compatriotes de ce dernier. Dotée en 1820 d'un équipement qu'on désigne du nom de " promenade des Anglais ", Nice constitue en quelque sorte le prototype des stations balnéaires créées à l'écart du rivage et des villages de pêcheurs. Connu grâce au peintre de marines Charles Mozin, le site de Trouville s'impose comme destination de villégiature après 1825. La multiplication des constructions y échappe toutefois à toute règle d'urbanisme et le site est rapidement saturé. Un projet d'extension est alors ébauché. II ne fait toutefois pas le poids face à un projet concurrent qui donne naissance à Deauville. Conçu à compter de la fin des les années 1850, le plan d'urbanisme ne vise pas qu'à ordonner en front de mer un voisinage de villas. II vise plutôt la fondation d'une 
ville nouvelle dotée d'infrastructures ferroviaires et portuaires et de grands équipements publics. Cette création est précédée de peu par la fondation de Cabourg-les-Bains, station dotée d'un plan en éventail, dont l'influence est perceptible à Deauville.

Portés par le courant hygiéniste, qui alimente la conception des nouveaux quartiers destinés aux classes privilégiées et les opérations de rénovation urbaine de la seconde moitié du XIXe siècle, ces projets de villes et de voisinages balnéaires européens constituent de véritables laboratoires d'urbanisme (Boyer, 2008), notamment en ce qui concerne l'organisation de la trame viaire, l'installation d'équipements infrastructurels et la profusion de verdure. Un peu partout, l'influence de l'urbanisme haussmannien et géorgien est manifeste. Grands hôtels, parcs, jardins, opéras, théâtres, pavillons de santé et casinos y polarisent les voisinages résidentiels et favorisent la sociabilité et l'étalage de la réussite des nantis, tandis que les gares ferroviaires rappellent que ces lieux constituent en quelque sorte des excroissances des capitales et des métropoles.

Passablement moins urbaines que leurs consœurs des vieux pays - un caractère distinctif au demeurant scrupuleusement préservé -, les stations du Nord-Est américain disséminées dans les provinces maritimes (St. Andrews), le bas Saint-Laurent (Rivièredu-Loup, Cacouna) et Charlevoix (Pointeau-Pic, La Malbaie, Saint-Irénée), ainsi que sur le littoral de la Nouvelle-Angleterre (Cape Cod, Ogunquit, Newport, Kennebunkport) partagent néanmoins avec celles-ci un même niveau de confort, de luxe et d'avancées technologiques. Quant à la Floride, elle entend proposer aux Américains des États du Nord, à compter de la toute fin du XIXe siècle, une alternative aux stations balnéaires européennes. La trajectoire évolutive des stations y suit, avec un décalage de quelques décennies, le modèle européen.

Même si l'engouement pour la montagne est contemporain de celui pour la mer, les stations aménagées en altitude n'en connaissent pas moins un développement plus tardif. Les premiers hôtels sont inaugurés un peu avant le milieu du XIX siècle. Ces stations sont essentiellement fréquentées durant l'été. On y vient pour l'air frais, les vues, les randonnées et, pour les plus téméraires, l'escalade. L'essor des sports d'hiver, en particulier le ski alpin, donne un second souffle à la montagne dès la fin du siècle et de véritables stations s'y développent.

\section{Les grandes stations contemporaines}

Le tournant des années 1950 aux années 1960 coïncide avec un accroissement de la mobilité des citoyens des pays industriels, un enrichissement de la classe moyenne, un accès étendu aux loisirs et une démocratisation du tourisme. Le tourisme de masse prend appui sur la construction de grands équipements d'hébergement, de sport et de loisirs, tant sur les littoraux qu'à la montagne. Alors que les hôtels s'étaient rapprochés du rivage et des promenades qui le longeaient à compter de la fin du XIX ${ }^{e}$ siècle, les grands ensembles s'y dressent, telles de véritables palissades. C'est désormais par dizaines de milliers que se comptent les unités d'hébergement groupées dans des barres, des tours et des constructions pyramidales, mais aussi parfois dans des unités de voisinage évoquant des habitats traditionnels. Dans un contexte de compétition parfois exacerbée, les plages et les domaines skiables ne suffisent toutefois plus à assurer la fréquentation. II faut désormais aménager des terrains de golf, construire des tennis, des piscines, des complexes aquatiques, des équipements multisports, des centres commerciaux, voire des centres de congrès, et moderniser les infrastructures de transport (aérien, ferroviaire et routier).

La géographie humaine et les écosystèmes naturels des milieux concernés sont radicalement bouleversés par la multiplication des installations et l'explosion du nombre des visiteurs. À tel point que les termes azurisation, baléarisation, marbelisation, riminisation et canarisation désignent désormais ces vastes opérations de bétonnage des fronts de mer. Maintenue à l'écart du dynamisme européen durant l'ère franquiste, l'Espagne est particulièrement touchée. En 2006, on y dénombrait dans les secteurs touristiques du littoral plus de 1,5 million d'appartements, plus de 300 golfs et plus d'une centaine de ports de plaisance en chantier ou planifiés. L'effet ne se fait toutefois pas sentir sur les seuls milieux d'accueil.
Déjà perceptible au moment du développement des premières stations balnéaires et de montagne, l'impact de ces équipements sur l'urbanisation s'est considérablement accru, à un point tel qu'il est parfois difficile d'établir une ligne de démarcation entre les deux phénomènes. Dans les années 1960, le néologisme villégiaturbanisation ${ }^{10}$ est incidemment forgé pour décrire l'interpénétration de la villégiature, supportée par des installations de sport de plein air et de loisirs et une périurbanisation embryonnaire dans les Basses-Laurentides, au nord de Montréal. De nos jours, le phénomène est extrêmement répandu, au point d'engendrer, sur bon nombre de littoraux de la planète, des corridors d'urbanisation à caractère récréotouristique quasi continus sur plusieurs centaines de kilomètres et sous-tendus par des pôles urbains plus ou moins anciens.

L'essor des stations thermales, puis de celles du littoral et de la montagne, s'inscrit dans le contexte d'une redéfinition de l'espace touristique que favorisent à des degrés divers l'insalubrité des villes, la montée de l'hygiénisme, ainsi que l'invention de la mer, de la montagne et de la campagne. Le phénomène a une ampleur telle qu'il suggère un abandon d'une référence urbaine (Boyer, 1996) qui avait dominé presque sans partage en Occident depuis la renaissance urbaine du Moyen Âge. À y regarder de plus près, on se rend toutefois compte qu'il s'agit davantage d'une mise entre parenthèses de la ville comme espace quasi exclusif d'une expérience culturelle que d'un abandon véritable de la référence urbaine.

Bath avait incidemment montré, dès sa renaissance au XVIII ${ }^{e}$ siècle, que les stations devaient en partie leur succès à un cadre bâti et à un environnement culturel résolument urbains. II n'en est pas autrement, on l'a également souligné, des grandes stations de la seconde moitié du XIX siècle. Certes, leur succès repose sur la mise à la disposition des visiteurs d'équipements dédiés, dont les grands hôtels, les casinos, les centres de santé et les salles de spectacles, mais plusieurs d'entre eux ne constituent justement pas des équipements spécifiquement touristiques, puisqu'on les retrouve également à la ville. Qui plus est, ils s'inscrivent habituellement dans un environnement bâti qui doit 
beaucoup à l'urbanisme du moment. Quant aux villes, en particulier les plus grandes, elles ne tardent pas à prendre leur revanche.

\section{Métropoles et capitales}

En deuxième moitié du XIX siècle et au début du $X X^{e}$, le développement des capitales européennes et des métropoles américaines est étroitement lié à l'essor du tourisme urbain. Londres et Paris donnent le ton. L'organisation d'expositions universelles y est notamment l'occasion de travaux qui marquent durablement l'imaginaire des visiteurs autant que le paysage urbain. Même si plusieurs constructions furent éphémères, un certain nombre d'équipements survivent aux événements et restent associés à l'activité touristique. Mais ces équipements sont indissociables de la ville qui les abrite.

Londres devient, au XIX ${ }^{e}$ siècle, la première ville millionnaire d'Occident depuis la Rome de l'Antiquité. La fondation du British Museum (1753) et celle de la National Gallery (1824) y avaient anticipé la mobilisation d'un imposant patrimoine culturel qui ferait la renommée de la ville. Mais le véritable coup d'envoi du positionnement de la capitale britannique sur la scène du tourisme est la tenue de la première exposition universelle de l'histoire, en 1851. L'immense palais de fonte et de verre érigé dans Hyde Park abrite les productions industrielles et artisanales présentées par plus de 13000 exposants provenant de toutes les parties de l'Empire et du reste du monde. Même si on y accueille seulement quelque 58500 visiteurs étrangers - Londres est déjà perçue comme une destination dispendieuse -, les revenus tirés des 4,5 millions d'entrées génèrent un profit qui est réinvesti dans un vaste complexe groupant des musées - Natural History Museum, Science Museum, Victoria \& Albert Museum -, des institutions d'enseignement - Royal College of Music, Imperial College - et une salle de spectacles - Royal Albert Hall - érigée dans South Kensington.

Des investissements importants ne sont toutefois pas consentis aux seules fins de la construction d'équipements culturels et de leur dotation en collections de tous genres. Le percement de Regent Street (1813-1827) et l'aménagement de Regent's Park (1818) donnent toute sa mesure au Londres de la période Régence. Les résidences de prestige se multiplient au gré de l'aménagement des squares et des avenues plantées de South Kensington, de Pimlico, de Belgravia et de Chelsea. Les galeries marchandes de St. James et de Mayfair et les grands magasins d'Oxford Street (Selfridges) et de Knightsbridge (Harrods) offrent de quoi satisfaire la clientèle la plus capricieuse. Whitehall et la City ne sont pas en reste. Gouvernement et institutions financières reconstruisent en effet dans un style monumental plusieurs des immeubles qui les logent. Londres est incontestablement la plus grande ville du monde et une destination touristique prisée.

Paris n'est pas en reste. Déjà, dans les années 1840, les rues de la Paix, de Castiglione et de Rivoli forment l'armature d'un « quartier londonien ». Puis, dans la deuxième moitié du siècle, des palaces et des passages accueillant des boutiques de luxe s'élèvent en bordure des Champs Élysées, faisant du quartier un des principaux lieux d'accueil des visiteurs et le centre cosmopolite de la vie parisienne (Vajda, 2007). Pendant qu'à Londres s'érigent les beaux quartiers du West End, à Paris, le baron Haussmann réalise de nombreuses percées, préside à l'érection d'immeubles de rapport et façonne le domaine public d'une manière qui va transformer durablement le visage de la capitale et en faire une référence urbaine. Les grandes expositions de 1867, 1878, 1889 et 1900 sont par ailleurs l'occasion de doter la ville de nombreux équipements - notamment des gares ferroviaires, des palais d'exposition, un aquarium, une première ligne de métro (1900), ainsi que la célèbre tour Eiffel (1889) - qui restent au service de la population, contribuant à consolider l'image de marque de la capitale et favorisant la venue de visiteurs.

Plusieurs autres villes participent à ce mouvement. Complètement transformée par l'urbanisme de Cerda en deuxième moitié du XIXe siècle, Barcelone tient une première exposition universelle en 1888 sur le site du parc de la Citadelle, aménagé en 1869. ॥ en reste quelques vestiges. L'exposition de 1929 laisse un héritage plus significatif. Le Palais national (musée d'art de la Catalogne), le village espagnol et le pavillon de l'Allemagne dessiné par Mies Van Der Rohe ${ }^{11}$ constituent en effet des éléments phares du secteur dominé par la colline Montjuic, un

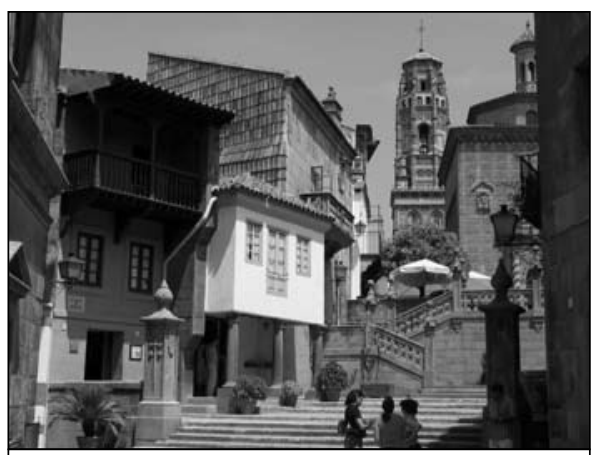

Illustration 3 : Le village espagnol de Barcelone a été construit à l'occasion de l'exposition universelle de 1929. II propose des reconstitutions de bâtiments traditionnels de différentes contrées

de l'Espagne.

Photo : G. Beaudet.

des principaux sites des Jeux olympiques de 1992. On y trouve le stade originellement construit en 1936 en vue de jeux qui devaient concurrencer ceux de Berlin, mais qui furent annulés en raison de la guerre civile.

L'Amérique participe aussi à ce concert des grandes villes désireuses de conquérir le monde. À la fin des années 1870, au moment où elle se relève de ses cendres, la ville de Chicago entend déclasser New York au titre de métropole étatsunienne. Cette rivalité trouve un écho dans la bataille que se livrent plusieurs villes en vue de la tenue de l'exposition colombienne prévue pour 1892 pour commémorer le $400^{\mathrm{e}}$ anniversaire de la venue de Christophe Colomb en Amérique. La «Ville des vents » l'emporte à l'arraché, mais doit reporter de un an l'inauguration de l'événement. Aménagé en parvis du centreville, où se dressent les premiers gratte-ciel de l'histoire, le site de l'exposition laisse une impression durable. On y signe en quelque sorte l'acte de naissance du mouvement City Beautiful. Influencé par le style néoclassique des principaux pavillons et des colonnades qui ceinturent une grandiose cour d'honneur au centre de laquelle avait été aménagé un vaste bassin, ce mouvement associe le renouveau civique à des arrangements urbains caractérisés par l'ordre et l'harmonie des agencements monumentaux.

Les villes qui se sont relayées après la Seconde Guerre mondiale pour organiser de telles expositions en profitent elles aussi pour mieux se faire connaître, de manière à pérenniser les 
retombées de l'événement, notamment d'un point de vue touristique. Pour ce faire, elles mettent en place des infrastructures et des équipements qui traduisent une volonté de modernisation et dont elles pourraient tirer profit par la suite. Encore évoquée dans la plupart des guides touristiques, l'exposition universelle organisée en 1958 par Bruxelles symbolise une telle volonté

d'affirmer son caractère ultramoderne. La notoriété de l'Expo 58, tout entière vouée au progrès technologique, rejaillit bien entendu sur la ville, d'autant que celle-ci en conserve l'une des réalisations les plus emblématiques, l'atomium. (Jaumain, 2007, p. 316)

La construction du métro et des îles du SaintLaurent - où fut tenue Expo 67 et où se dresse toujours la structure de la sphère du pavillon étatsunien dessiné par Buckminster Fuller - joue un rôle similaire pour Montréal. ॥ en avait été de même en 1962 pour Seattle où la tour connue sous le nom de Space Needle et le parc thématique qui se déploie à sa base anticipaient l'entrée dans le XXle siècle. Quant à Lisbonne, elle profite notamment de l'exposition de 1998 pour prolonger et réorganiser les circuits de son métro.

\section{Les installations olympiques}

La naissance des Jeux olympiques modernes, organisés à Athènes en 1896, confère peu à peu aux activités sportives un prestige qui avait jusque-là été surtout l'apanage de la culture, de la science et de l'innovation technologique. Comme les expositions universelles et internationales, les grands événements sportifs (Jeux olympiques d'été, puis d'hiver, Mondiaux de soccer, Jeux du Commonwealth, Jeux panaméricains, etc.) mobilisent la participation de plusieurs pays, favorisent la venue de nombreux visiteurs et sont pour les villes hôtes, particulièrement à compter des années 1950, un outil de marketing aux retombées de plus en plus considérables. Mais, comme l'était depuis un demi-siècle l'organisation des grandes expositions, leur tenue est également prétexte à d'importantes opérations urbanistiques et immobilières, dont on cherche à optimiser les retombées.

L'Europe et les États-Unis restent le terrain d'expérimentation des Jeux olympiques jusqu'en 1952 pour les Jeux d'été et jusqu'en 1972 pour ceux d'hiver. Les premiers jeux sont des événements passablement modestes et ils ont un impact limité. Ceux de Paris (1900) et de St. Louis (1904) sont d'ailleurs couplés à des expositions universelles, tandis que ceux de Londres (1908) le sont à une exposition franco-britannique. Mais, à compter de 1912 (Stockholm), les jeux sont tenus de manière autonome. Bien que les organisateurs des Jeux de Paris aient souhaité, en vain, faire profiter l'Est parisien de leur tenue, la principale retombée reste, jusqu'en 1924, outre la venue de visiteurs, la construction d'un stade principal capable d'accueillir par la suite de nombreux événements sportifs locaux, nationaux et internationaux, ce qui n'est pas banal en ces années où le tourisme reste encore affaire, pour beaucoup, sinon des privilégiés du moins des membres des classes supérieures, et où une véritable culture de masse du sport se développe.

Si les villes savent miser sur l'organisation de ces événements sportifs pour se doter de nouveaux équipements et réaliser certains travaux d'urbanisme - à Paris, en 1924, on innove en construisant le premier village olympique, qui sera transformé en logements après les jeux -, il revient à Rome et à Berlin de changer radicalement la donne.

Ayant accédé au pouvoir en 1922, à la suite de la marche des fascistes sur Rome, Mussolini entretient pour la capitale des projets grandioses. Le culte voué par le mouvement fasciste aux sports et aux athlètes l'incite à mettre en œuvre, dès 1928, un ambitieux projet de construction d'un complexe sportif destiné à accueillir d'éventuelles compétitions de grande envergure et à glorifier le Régime. Le complexe du Foro Mussolini (devenu Foro Italica après la Seconde Guerre mondiale) nécessite le drainage d'une plaine marécageuse en rive droite du Tibre, à plus de un kilomètre au nord-est du Vatican. Le complexe comprend un stade de 16000 places, des thermes, deux piscines, une académie d'escrime, une palestre, ainsi que des courts de tennis. Une vaste esplanade débouchant sur un pont complète les aménagements. Inachevé à la mort du duce, le complexe est le cœur des installations destinées à abriter les Jeux olympiques de 1960 à la suite de l'érection d'un stade de 80000 places.
Ce complexe n'est qu'une des composantes d'un projet urbain destiné à réaffirmer la grandeur de Rome. Mussolini commande en effet des travaux voués à éradiquer des voisinages de logements insalubres et à élargir certaines voies de circulation. Mais il table également sur la tenue d'une exposition universelle dont l'organisation avait été confiée à la capitale en 1936. Sur un emplacement de quelque 470 hectares situé au sud de la ville, le dictateur lance le chantier d'un quartier destiné à désengorger le centre de la ville. Identifié par l'acronyme EUR (Esposizione Universale Roma), le projet entend renouer avec la grandeur de la Rome impériale, tout en s'inspirant du futurisme italien. En bordure de larges avenues, on trouve notamment un pavillon du travail (Palazzo della Civilita del Lavoro), un pavillon de la science et un palais des congrès. Le déclenchement de la Seconde Guerre mondiale ne permet toutefois pas l'achèvement du projet originel et la tenue de l'exposition, prévue pour 1942. Le quartier, complété dans les années 1950 et abritant plusieurs musées, est aujourd'hui fréquenté par de nombreux visiteurs. Les deux réalisations, quoique entachées par leur origine fasciste, n'en constituent pas moins une anticipation de la manière dont les municipalités souhaitent capitaliser sur de tels événements d'envergure pour attirer les visiteurs, doter les villes hôtes de nouveaux équipements et consolider les assises de leur développement touristique.

L'Allemagne nazie non plus ne serait pas en reste. Berlin avait été désignée pour tenir les jeux de 1916, mais ceux-ci avaient été annulés en raison de la guerre. Elle avait à nouveau été choisie en 1931 en prévision des jeux de 1936. L'accession au pouvoir d'Adolphe Hitler, en 1933, conféra toutefois à l'événement une dimension nouvelle, notamment en raison du culte voué par les idéologues nazis au sport. Hitler voulait faire des jeux une vitrine du national-socialisme et de la grandeur arienne. Mais il souhaitait surtout faire de Berlin - qui serait renommée Germania - une capitale à la mesure des ambitions du troisième Reich. Concurremment à l'érection des installations sportives groupées dans un vaste parc autour d'un stade de quelque 100000 places et d'un village des athlètes, le projet confié à l'architecte Albert Speer ne 
prévoyait rien de moins que la reconstruction de la ville. L'élargissement de la principale avenue de Berlin constituait une des pièces maîtresses de l'opération. On en ferait une voie monumentale s'étirant sur cinq kilomètres et dont les extrémités seraient respectivement marquées par un immense édifice voûté de 250 mètres de diamètre et par un gigantesque arc de triomphe. Plusieurs bâtiments publics d'architecture néoclassique devaient compléter cette composition, dont une nouvelle chancellerie, qui comporterait un hall deux fois plus long que la galerie des Glaces de Versailles. La reconstruction de la ville devait être achevée à temps pour la tenue d'une exposition universelle projetée en 1950. La guerre interrompit toutefois les travaux. Outre les installations olympiques, seuls ont survécu aux destructions massives des derniers mois de la guerre l'aéroport de Tempelhof, un quartier de casernes d'une superficie de 80 hectares, ainsi qu'un complexe administratif.

La démesure du projet hitlérien et le désastre consécutif à l'effondrement de l'Allemagne nazie ne diminuent pas l'intérêt pour la tenue de grands événements sportifs qui peuvent être mobilisés dans le contexte de projets de renouvellement urbain. Les 16 villes hôtes des jeux depuis 1948 réalisent en effet toutes, bien qu'à des degrés divers, des grands travaux d'infrastructure et se dotent d'équipements susceptibles de favoriser le développement et la diffusion d'une image de marque. De ce point de vue, Pékin semble établir un nouveau cadre de référence, tant en raison de la taille des équipements mis en place que par l'envergure des réaménagements urbains, réalisés souvent au détriment des cadres bâtis traditionnels et de leurs populations.

À la différence des Jeux olympiques d'été, les Jeux d'hiver sont d'abord l'affaire de stations de sports d'hiver, de Chamonix (1924) à Squaw Valley (1960), exception faite des jeux de 1952 qui sont organisés par la ville d'Oslo. II s'agit, pour l'essentiel, de consolider et de diversifier le parc des installations sportives, tout en augmentant la capacité d'hébergement, sans oublier l'impact d'un marketing susceptible d'engendrer des retombées durables en termes de fréquentation.
À compter de 1964 (Innsbruck) - à l'exception de 1980 (Lake Placid) -, les Jeux olympiques d'hiver sont organisés par des petites villes (Albertville et Lillehammer), mais surtout par des villes de taille moyenne (Grenoble, Sarajevo, Nagano) et des grandes villes (Sapporo, Calgary, Salt Lake City, Turin, Vancouver). Comme les ressources mobilisables - en l'occurrence la montagne - ne sont généralement pas localisées au cœur de l'agglomération, les stratégies qui président au déploiement des installations sont désormais d'échelle régionale et la mise en place d'infrastructures (aéroport, voies ferrées, routes et autoroutes, réseaux de télécommunication, etc.) accapare une part significative des investissements (Anonyme, 2008). L'accroissement anticipé du tourisme y est moins vu comme une fin en soi que comme une consécration du repositionnement de la ville sur l'échiquier des villes d'envergure internationale.

\section{Conclusion}

\|l ressort de ce survol que l'urbanisation qu'ont connue la seconde moitié du XIX' siècle et l'ensemble du XX siècle peut difficilement être pensée sans qu'on fasse référence au rôle qu'a joué le tourisme, particulièrement dans le cas des métropoles et des capitales, d'une part, et des stations touristiques, d'autre part. Le phénomène est à nouveau avéré depuis deux décennies avec l'arrivée de plusieurs villes de tailles diverses sur l'échiquier du tourisme.

En effet, depuis le tournant des années 1980 aux années 1990, le repositionnement vocationnel de nombreuses villes éprouvées par la désindustrialisation s'articule explicitement à la culture, au loisir et au sport. Plusieurs équipements d'envergure, dont, en particulier, des centres de congrès, des stades et des musées, signés par les plus grands noms de l'architecture, y incarnent avec panache ce renouveau. Ce faisant, ces villes ont toutes cherché à consolider leur attractivité touristique, même si le tourisme n'est pas la vocation première de bon nombre d'entre elles.

En fait, comme le montrent de nombreux exemples, dont celui de Barcelone (Olagnier, 2007), le tourisme est plus que jamais intimement lié à l'évolution des villes et à la transformation de leur centre aussi bien que de l'ensemble de leurs quartiers anciens. II est tout simplement devenu un attribut essentiel des grandes villes (Violier et Zarate, 2007).

La ville, et en premier lieu la métropole, fonde son fonctionnement touristique sur trois ressorts principaux : la redécouverte du patrimoine, le goût pour la modernité qui ne s'est presque jamais démenti et qui, à nouveau, s'exprime dans les grandes réalisations architecturales et urbanistiques, enfin, la participation à de grands événements qui permettent d'alimenter cette modernité urbaine et aux touristes de partager lieux et moments de la vie de la ville. (Knafou, 2007 : 10)

La ville n'est conséquemment pas que le réceptacle d'une fréquentation touristique qui serait mobilisée par quelques équipements spécifiques, même si les grands musées, les centres de congrès, les stades et d'autres installations prestigieuses signés par les plus grands noms de l'architecture y incarnent depuis quelques années avec panache un renouveau urbain fortement articulé au tourisme. Elle constituerait plutôt, ainsi qu'on l'a suggéré d'entrée de jeu, un hyperéquipement, dans la mesure où chacune de ses composantes est susceptible de constituer une ressource appropriable, d'une manière ou d'une autre, seule ou en synergie, par le tourisme. L'organisation d'événements de grande renommée - dont les Jeux olympiques et les expositions universelles -, comme la réalisation de grands projets qui leurs sont associés, sont néanmoins au cœur des stratégies de leur positionnement marketing, autant que de celles de leur réaménagement. Mais elles n'y trouvent pas leur fondement exclusif.

Les mises en scène qui se sont multipliées ces dernières années - parfois à l'échelle d'une agglomération, comme le montrent les plans Lumière territoriaux de Lyon - révèlent en effet le degré d'intégration de l'ensemble des composantes urbaines aptes à générer un potentiel d'appropriation touristique. Le déploiement de dispositifs techniques, technologiques, informationnels et scéniques destinés à supporter des activités et des événements artistiques, culturels et festifs dans le cadre de l'opération Lille 2004, capitale européenne de la culture en constitue un autre exemple (Collin, 2007). Ce déploiement 
s'inscrivait dans le sillage des investissements massifs en infrastructures et en équipements mis en œuvre par la ville depuis le début des années 1990 après l'adoption d'une politique de renouvellement urbain.

Le survol proposé dans cet article suggère que les hyperéquipements du tourisme sont autant le produit des adaptations de la ville au phénomène touristique que des contributions de l'urbanisme à l'évolution du tourisme. Une rupture d'échelle semble cependant sur le point de se produire. Plusieurs des exemples évoqués apparaissent en effet bien modestes quand on les apprécie à l'aune des opérations immobilières lancées à Pékin en vue des Jeux de l'été 2008 et de celles de Dubaï. Les premières ont incidemment été dénoncées par plusieurs observateurs, certains les ayant même comparées, par leur démesure et leurs conséquences, au projet nazi de reconstruction de Berlin (Parra, 2007). Quant à celles qui ont cours sur les rives du golfe Persique, elles ont été assimilées par Davis (2007) au résultat d'une convocation d'Albert Speer et de Walt Disney sur un terrain soumis à la fois à un absolutisme féodal et à un capitalisme sauvage qui n'aurait rien à envier à sa contrepartie du XIXe siècle. En un moment où l'on commence à peine à mesurer dans toute leur ampleur les impacts environnementaux et sociaux du développement des grandes installations touristiques de la seconde moitié du siècle dernier, n'y a-t-il pas lieu de s'inquiéter de l'émergence de tels hyperéquipements ?

Gérard Beaudet est directeur de l'Institut d'urbanisme de la Faculté de l'aménagement à l'Université de Montréal.

\section{Notes}

1 Et, de plus en plus, d'un système d'enneigement artificiel, y compris dans les stations de haute montagne.

2 Le peintre et lithographe Jules Chéret (18321932) est un des premiers propagandistes de l'application de la lithographie en couleur à la promotion des destinations touristiques desservies par les compagnies de chemin de fer (Caracalla, 2005). Au Canada, le C.P. utilise également l'affiche à cette fin à compter du début des années 1880 (Choko et Jones, 1988).

3 Un itinéraire alternatif est inauguré en 1919 par l'Italie après le percement du tunnel du Simplon. Le Simplon-Orient-Express reliait Calais - avec correspondance en provenance
d'Angleterre - à Constantinople et Athènes, en passant par Paris, Lausanne, Milan, Vérone, Venise, Trieste, Zagreb, Belgrade, Sofia et Salonique.

4 On favorise aussi la perpétuation de savoirfaire en créant un marché pour la restauration de barges anciennes et la construction de barges s'inspirant des embarcations traditionnelles.

5 La veduta est une toile habituellement de grand format présentant dans le menu détail un panorama urbain ou un paysage de campagne.

6 En évoquant le Paris médiéval dans son roman Notre-Dame-de-Paris, Victor Hugo est du nombre des précurseurs d'une valorisation de l'architecture vernaculaire et des formes urbaines modestes. II ne semble toutefois pas s'être opposé aux transformations qui avaient cours dans Paris. Quelques années plus tard, l'écrivain britannique John Ruskin fait l'apologie des tissus mineurs hérités du Moyen Âge. Ses propos trouvent toutefois peu d'échos chez les spécialistes de la conservation et encore moins chez les touristes de l'époque.

7 Bien que fréquentée dès les années 1860, Carcassonne ne peut compter sur un hôtel de prestige qu'à compter de 1909, au moment où est inauguré l'Hôtel de la Cité.

8 Le Mexique, ainsi que les Amériques centrale et du Sud, livrent aussi leurs impressionnants vestiges archéologiques à compter de la fin du XVIII e siècle et au cours de la première moitié du XIX - par exemple les cités mayas de Palenque (1807), d'Uxmal (1838) et de Tikal (1848) - ; les difficultés d'accès considérables les maintiennent toutefois longtemps à l'écart des principales destinations touristiques.

9 Organisation des Nations Unies pour l'éducation, la science et la culture.

10 Le terme est créé par le géographe aménagiste Jean Décarie à l'occasion d'une étude d'urbanisme réalisée dans la région de SaintSauveur, récemment desservie par l'autoroute des Laurentides.

11 Le pavillon avait été démonté à la fin de l'exposition ; reconnu comme une des icônes de l'architecture moderne, il est reconstruit en 1995.

\section{Bibliographie}

Adam, Robert (1764) Ruins of the Palace of the Emperor Diocletian at Spalatro in Dalmatia London, [http://digital.library.wisc.edu/1711. dl/DLDecArts.AdamRuins], consulté le 16 mai 2008.

Anonyme (2008), " Les villes candidates et organisatrices des Jeux olympiques d'hiver "»", [http://multimedia.olympic.org/pdf/ fr_report_666.pdf], consulté le 16 mai 2008.

Boyer, Marc (1996), L'invention du tourisme, Paris, Découvertes Gallimard.

Boyer, Marc (2008) Les villégiatures $d u X V^{e}$ au XXI siècle; panorama du tourisme sédentaire, Colombelles, Éditions EMS.

Caracalla, Jean-Paul (2005), Le voyage s'affiche, Paris, Fitway.
Choko, Marc et David L. Jones (1988), Canadien Pacifique, Affiches 1883-1963, Montréal, Éditions du Méridien.

Collin, Isabelle (2007), " De l'événement au tourisme au cœur de la métropole Lille 2004 capitale européenne de la culture ", dans Philippe Duhamel et Rémy Knafou (dir.), Mondes urbains du tourisme, Paris, Belin, p. 103-115.

Cossic, Annick (2003), "Une ville d'eaux à la mode au $18^{\mathrm{e}}$ siècle : la dialectique de la rupture et de la continuité à Bath ", Les cahiers du CEIMA (Centre d'études interdisciplinaires du monde anglophone), n 1, p. 19-35.

Davis, Mike (2007), Le stade Dubaï du capitalisme, Paris, Éditions des Prairies ordinaires.

Denon, Vivant (1802 [rééd. 2007]), Voyage dans la haute et basse Égypte pendant les campagnes du général Bonaparte, Paris, Éditions Pygmalion.

Gelbman, Alon (2007), "Tourism in Industry in the Post-industrial City ", dans Philippe Duhamel et Rémy Knafou (dir.), Mondes urbains du tourisme, Paris, Belin, p. 151-162.

Jaumain, Serge (2007), "'Une grande capitale moderne'. Bruxelles dans les guides touristiques de la seconde moitié du XXe siècle ", dans Philippe Duhamel et Rémy Knafou (dir.), Mondes urbains du tourisme, Paris, Belin, p. 308-322.

Jeancolas, Claude (1999), Venise et ses peintres ; une histoire intime, Paris, Éditions Van Wilder.

Knafou, Rémy (2007), "L'urbain et le tourisme : une construction laborieuse ", dans Philippe Duhamel et Rémy Knafou (dir.), Mondes urbains du tourisme, Paris, Belin, p. 9-21.

Lambert, Robin (2001), Patrimoine et identité : Bath 1900-1960, Rennes, Presses universitaires de Rennes.

Olagnier, Pierre-Jacques (2007), " Le tourisme, instrument pertinent des politiques urbaines? Le cas de Barcelone ", dans Philippe Duhamel et Rémy Knafou (dir.), Mondes urbains du tourisme, Paris, Belin, p. 176-187.

Parra, Aritz (2007), « Urbanisme 'Nazi' à Pékin », Courrier international, [http://www.courrierinternational.com/article.asp?obj_id=76562], consulté le 15 août 2007.

Saurat, Catherine (1999), Villes d'eaux en Europe, Paris, Éditions du Chêne.

Vajda, Joanne (2007), " L'élite voyageuse et la transformation de l'espace parisien, 18551937 ", dans Philippe Duhamel et Rémy Knafou (dir.), Mondes urbains du tourisme, Paris, Belin, p. 116-127.

Violier, Philippe et Antonio Manuel Zarate (2007), "Politiques urbaines du tourisme ", dans Philippe Duhamel et Rémy Knafou (dir.), Mondes urbains du tourisme, Paris, Belin, p. $143-162$

Voltaire (1749) Des embellissements de Paris, [http://www.voltaire-integral.com/Html/23/ 30Embellissements.htlm], consulté le 16 mai 2008.

Wackermann, Gabriel (1988), Le tourisme international, Paris, Armand Colin. 